\title{
Tutoria nas aulas de educação física inclusiva: uma revisão sistemática
}

\author{
Tutoring in inclusive physical education classes: a systematic review
}

Juliana Aparecida de Paula Schuller, ${ }^{1,2}$, Cléria Maria Lobo Bittar, ${ }^{2}$ Lilian Cristina Gomes do Nascimento, ${ }^{2}$ Maysa Venturoso Gongora Buckeridge Serra, ${ }^{2}$ Maria Georgina Marques Tonello²

'Universidade Federal de Mato Grosso, Cuiabá, MT, Brasil.

ZUniversidade de Franca, Franca, SP, Brasil.

Recebido em: 11/08/2016 / Aceito em: 02/09/2016 / Publicado em: 30/09/2016

juliana_schuller@yahoo.com.br

\section{RESUMO}

Para que as aulas de educação física sejam efetivamente inclusivas, uma estratégia que vem sendo adotada por profissionais da área é a tutoria. Objetivo: realizou-se uma revisão sistemática da literatura sobre os efeitos da tutoria na inclusão de alunos com deficiência nas aulas de educação física. Método: foi realizada uma busca de artigos nas bases Periódico da Capes e Web of Knowledge, com os termos: "Peer Tutoring e "Physical Education". Foram incluídos artigos que tivessem a tutoria como estratégia usada na inclusão de alunos com deficiência nas aulas de educação física, selecionando quatro artigos. Resultados: verificou-se que a inclusão de alunos com deficiência nas aulas de educação física pode não ser bem-sucedida, caso não haja uma assistência complementar oferecida aos alunos com deficiência nas atividades desenvolvidas. Considerações finais: o uso da tutoria de pares por tutores de mesma idade das pessoas com deficiência mostra-se uma valiosa estratégia para a inclusão, pois esta favorece positivamente interações entre os alunos.

Palavras-chave: Inclusão; Educação Especial; Diretos das pessoas com deficiência.

\section{ABSTRACT}

A strategy that has been adopted by physical education professionals in order to make the physical education classes effectively inclusive is the tutoring. Objetive: A systematic literature review about the effects of tutoring in the inclusion of challenged students in the physical education classes was done. Method: An article research on the Capes periodical basis and Web of knowledge was done, with the following terms:
"Peer tutoring" and "Physical Education". Articles that had tutoring as a strategy used in the inclusion of challenged students in the physical education classes were included, four articles were selected. Results: It was verified that the inclusion of challenged students in the physical education classes may not be well succeeded, if there is no complementary assistance offered to the challenged students in the activities developed. Closing remarks: The use of peer tutoring for tutors from the same age group of the challenged people shows itself as a rich strategy for the inclusion, because it stimulates positively the interactions among students.

Keywords: Inclusion; Special education; Direct people with disabilities.

\section{INTRODUÇÃO}

A deficiência tem sido, desde a era moderna, interpretada pela visão da biologia e da medicina, fato este que define a maneira como a interpretamos. Entretanto, a deficiência é tão socialmente definida que ainda nos dias atuais, as palavras que mais expressam a opinião acerca dela estão associadas à limitação e superação. ${ }^{1}$ A limitação da pessoa com deficiência está associada a uma suposta condição de dependência física, social e afetiva das pessoas ao seu redor e uma frequente necessidade de superação dos obstáculos. Esta visão coordena a concepção de que as pessoas com deficiência devem transcender sua condição para que possam inserir-se nas mais distintas relações sociais.

O modelo médico aborda a deficiência por meio de um conjunto de teorias e práticas assistenciais, que pressupõe relação de causalidade entre a lesão ou a doença e a experiência da deficiência. ${ }^{2}$ Assim, as questões relacionadas à deficiência incluem situações nor- 
malmente associadas a ela, tais como, as condições de limitação/incapacidade, segregação/exclusão e situações de vulnerabilidade que frequentemente acompanham a pessoa que difere da grande maioria que os cerca.

Para que condições relacionadas à saúde e educação sejam propiciadas a todos os diferentes grupos sociais, incluindo os grupos vulneráveis: pessoas com deficiências, o princípio de equidade e justiça social devem ser pré-requisitos para a defesa da saúde e educação. ${ }^{3}$ Para muitos, parece ser incompatível uma pessoa com deficiência apresentar uma boa condição de saúde e qualidade de vida. Por outro lado, o reconhecimento da capacidade de desenvolvimento integral da pessoa com deficiência, significa abrir espaços para o empoderamento, nas suas escolhas de vida e na busca por oportunidades educacionais, ocupacionais e de lazer. ${ }^{4}$

A pessoa com deficiência ainda pode carregar consigo todo um estigma marcado por uma condição: a diferença. $O$ diferente ainda amedronta e a consequência do medo, aliado ao desconhecimento acerca da deficiência, ainda gera o preconceito, a discriminação e a exclusão, que colocam a pessoa com deficiência numa condição de vulnerabilidade. De modo geral, as pessoas com deficiência são consideradas dependentes dos cuidados de outros, incapazes de estudar e trabaIhar, isentas dos deveres normais e, acredita-se, necessitadas de cura, tratamento, reabilitação e habilitação. ${ }^{5}$

No contexto escolar, a realidade da pessoa com deficiência não difere da visão apresentada nos demais contextos sociais. No Brasil, nas décadas de 1980 e 1990 , os governos estaduais e federais tomaram iniciativas para modernizar as leis relacionadas aos direitos das pessoas com deficiências. ${ }^{6}$ Entretanto, apesar dos direitos de acesso à educação no ensino regular, estabelecidos em Declarações, Leis e Decretos, como: Constituição Federal do Brasil - 1988; Declaração Mundial sobre Educação para todos - 1990; Declaração de Salamanca - 1994; Lei de Diretrizes e Bases - 1996; Lei n. 9.394 - 1996; Resolução CNE/CEB n02/2001; Política Nacional de Educação Especial na Perspectiva da Educação Inclusiva - 2008; Lei n. 11.494 - 2007,7-9 dentre outras. O que ainda temos nos dias de hoje é uma batalha constante em oportunizar as pessoas com deficiência, o acesso à educação de qualidade.

$\mathrm{O}$ aumento de alunos com deficiência matriculados na rede regular de ensino é significativo e aponta para uma necessidade de que, cada vez mais, as escolas, os professores e os alunos, de um modo geral, estejam preparados para lidar com este panorama diferenciado. Onde a diferença não será mais um fator isolado e sim em uma realidade permanente que oportunizará a todos, o aprendizado que vai além dos conteúdos ministrados, ou seja, a oportunidade de conhecer o diferente.

Para Soler ${ }^{10}$ a inclusão é uma grande oportunidade para a transformação do ambiente escolar, e também uma chance do aprimoramento dos professores, investindo em formação, para que assim possam lidar com a diversidade, visando uma nova escola e, consequentemente, uma nova sociedade. Quando refletimos a respeito da inclusão escolar percebemos que existe uma complexidade permeando todo esse processo, as políticas econômicas e educacionais, a forma que está sendo implantada, a formação dos professores, as barreiras atitudinais como os preconceitos, os estigmas, a não aceitação das diferenças, dentre outros. ${ }^{11}$

Embora a legislação brasileira garanta o acesso e a permanência de estudantes com deficiências na rede regular de ensino, observa-se que a inclusão ainda é uma realidade ambígua no contexto escolar, repetindo-se o mesmo panorama nas aulas de educação física. No Brasil, muitos estudantes com deficiências permanecem excluídos ou tem sua participação limitada nas aulas de educação física, embora esta seja um componente curricular obrigatório em diferentes níveis do ensino básico. ${ }^{12}$

A educação física, como um dos componentes curriculares da educação básica, não pode ficar indiferente ou neutra frente ao movimento da educação inclusiva. Nessa disciplina deve-se estabelecer como um dos assessores do processo da inclusão escolar e social. ${ }^{13}$ A participação nas aulas de educação física pode modificar estilos de vida que determinam a saúde de indivíduos com deficiências. Dos avanços advindos na área da promoção de saúde destacam-se as mudanças comportamentais, tais como as mudanças de estilo de vida, escolhas saudáveis, alimentação adequada, atividade física regular, lazer ativo, redução do stress. As tendências demográficas tais como a urbanização, um comportamento mais sedentário, ameaçam a saúde e o bem-estar de centenas de milhões de pessoas. ${ }^{14}$

Desta forma, o processo de inclusão torna-se imprescindível para promover a participação dos alunos com deficiências nas aulas de educação física e a prática de seu amplo conteúdo. Todavia, ainda existem limitações que dificultam este processo, tais como a falta de conhecimento do profissional acerca das deficiências, e também uma carência em sua formação que o permitam adotar estratégias diferenciadas, quanto à elaboração dos conteúdos a serem trabalhados em suas aulas, os quais realmente desempenhem de um modo a propiciar a real inclusão.

Neste sentido, e de modo a garantir que as aulas de educação física sejam efetivamente inclusivas, uma das estratégias que vem sendo adotadas e incentivadas por profissionais da área é a utilização da tutoria. Em uma revisão sistemática realizada por Qi e Há, ${ }^{15}$ sobre a inclusão nas aulas de educação física no período de 1990 a 2009, foram encontrados 75 artigos que abordavam esta temática. Os resultados mostraram que as publicações têm aumentado ao longo dos anos e que três temáticas são recorrentes: partes interessadas na perspectiva inclusiva (65\%), práticas inclusivas eficazes $(12 \%)$ e os impactos da inclusão nos alunos com e sem deficiência (19\%). Os estudos sugerem que estratégias, tais como a tutoria entre os pares, podem ser um apoio útil na educação física inclusiva.

A tutoria é uma das estratégias de ensino em que o professor prepara um colega da mesma sala de aula ou da escola do aluno com deficiência para auxiliá-lo na execução das atividades. Esse tipo de estratégia, além de propiciar condições para a inclusão, também contribui no processo de empoderamento das pessoas com deficiências e em sua participação efetiva nas aulas de Educação Física. Desta forma, a adoção desta estratégia admite que no modelo inclusivo, ambas as partes aprendam e ensinem a si próprias, ao outro e ao professor, e ainda estimula a todos a reconhecerem o valor da diversidade. ${ }^{16-18}$

Repensando a prática da educação física e a 
necessidade de estratégias de ensino que favoreçam o processo de inclusão, o objetivo desta pesquisa foi realizar uma revisão sistemática bibliográfica, buscando trabalhos que analisaram os efeitos da tutoria na inclusão de alunos com deficiência nas aulas de educação física.

\section{DESENVOLVIMENTO}

A busca da melhoraria da qualidade das ações na saúde e no ensino refletiu na forma como é realizada a seleção e compilação dos estudos a serem utilizados como fontes na pesquisa. Se antes a consulta de artigos priorizava os estudos primários, atualmente, em decorrência do grande número de produções com a mesma temática, utiliza-se da realização de revisões sistemáticas no intuito de captar, reconhecer e sintetizar as evidências científicas. ${ }^{19}$

Os critérios utilizados para inclusão dos artigos nesta revisão foram as publicações que se utilizaram da tutoria como estratégia de ensino para inclusão de alunos com deficiência nas aulas de educação física, os quais tivemos acesso ao artigo completo. Os critérios de exclusão utilizados foram aplicados para as publicações que usaram da tutoria fora do contexto das aulas de educação física, tais como o esporte de rendimento, ou ainda os estudos que não tivessem como participantes os alunos com deficiências.

Neste estudo utilizamos de um método sistemático para realização do mesmo, onde, numa primeira fase foi previamente consultado na página online dos Descritores em Ciências da Saúde (DeCS), os termos de busca adequados para esta pesquisa, entretanto não foi encontrado nenhum termo equivalente à "Tutoria" ou "Tutoria aos Pares". Assim, optou-se por adotar o termo "Peer Tutoring", já que o mesmo é comumente utilizado na literatura atual. O segundo termo utilizado foi "Physical Education", definido como: programa de instruções para o cuidado e desenvolvimento do corpo, frequentemente em escolas.

Para a construção deste estudo, buscou-se artigos nas seguintes fontes de busca: Periódico Capes e Web of Science, utilizando do seguinte cruzamento em busca avançada: "Peer Tutoring" and "Physical Education". A partir da busca, segundo os critérios pré-estabelecidos, encontrou-se um total de 118 artigos elegíveis (Periódico da Capes $=84$ e no Web of Knowledge $=34$ artigos). Foram aplicados os seguintes filtros nesta etapa: documentos cujos periódicos publicados fossem revisados por pares; documentos publicados entre os anos de 2005 e 2015; que fossem publicados nos idiomas inglês, português ou espanhol; artigos completos (excluindo assim, resenhas, atas de congressos, artigos de jornais, ou outros). Após a aplicação destes filtros, atingiu-se um total de 58 artigos (Periódico da Capes = 48 e no Web of Knowledge $=10$ artigos).

Num segundo momento, foram analisados os títulos dos artigos elegíveis, conforme os critérios de inclusão, e a partir desta verificação excluiram-se os títulos duplicados (13), os textos que não possuíam resumo/abstract (nove), passando assim a trabalhar com 36 documentos elegíveis para a próxima fase desta revisão, que foi a leitura dos resumos.

Após seleção dos resumos, os estudos que aten- deram os critérios de inclusão e exclusão foram lidos na íntegra. Nesta etapa, a revisão foi efetuada independentemente por dois pesquisadores, ao final, quatro artigos atenderam a todos os critérios de inclusão desta pesquisa. Na tabela 01 estão representadas as etapas para a seleção dos artigos.

Tabela 1 - Percurso de seleção dos artigos incluídos na presente revisão.

\begin{tabular}{lcc}
\hline & \multicolumn{2}{c}{ Base de dados pesquisada } \\
\cline { 2 - 3 } & Periódico da & Web of \\
\cline { 2 - 3 } & Capes & Knowledge \\
\hline Total de artigos & 84 & 34 \\
Total de artigos excluídos & & 118 \\
$\quad$-Periódicos não revisados por pares & & \\
-Publicações anteriores a 2005 & 07 & - \\
-Não publicados em português, inglês ou & 23 & 08 \\
espanhol & - & \\
-Recursos, textuais, resenhas, atas) & 06 & 11 \\
-Artigos duplicados nas bases & & 05 \\
-Artigos sem resumo & & 13 \\
-Artigos com temas incompatíveis & & 09 \\
$\quad$ ao objetivo da revisão & & 32 \\
\hline Total de artigos selecionados & & 04 \\
\hline
\end{tabular}

$\mathrm{Na}$ avaliação dos artigos, foram considerados os seguintes aspectos: autores, ano de publicação, objetivos apresentados, participantes, metodologia utilizada e resultados/conclusões obtidas (Quadro 01).

A presente revisão sistemática selecionou quatro artigos, ${ }^{20-23}$ os quais apresentaram resultados relacionados sobre a tutoria em alunos com deficiência nas aulas de educação física. Através destes achados, podemos observar a escassez de publicações nacionais sobre esta temática, visto que todos os estudos selecionados são internacionais, dos quais três estudos são procedentes dos Estados Unidos e um da Suécia.

A amostra dos artigos selecionados foi composta por um total de 75 participantes, entre tutorados e tutores, dos quais 15 apresentavam algum tipo de deficiência e 60 não apresentavam deficiências. As deficiências foco dos estudos foram: deficiências múltiplas, deficiência física, deficiência intelectual leve e moderada, autismo, deficiência visual e baixa visão. A faixa etária dos estudantes avaliados variou entre 8 e 16 anos. Com relação ao tamanho da amostra utilizada pelos pesquisadores dos estudos selecionados, notou-se uma variação entre 3 e 4 para alunos com deficiência e de 4 a 37 para alunos sem deficiência, com média amostral total de 19 participantes $( \pm 15,04 \mathrm{DP})$ por estudo. Vale ressaltar que nem todos os autores trouxeram explicito o sexo dos participantes da pesquisa, com e sem deficiência.

Dos artigos selecionados identificou-se que os objetivos principais são: a interação comportamental dos alunos com deficiência, alunos sem deficiências e professores nas aulas de educação física, o tempo de aprendizado acadêmico nas aulas de educação física de alunos com e sem deficiência, e modificações no nível de habilidades motoras dos alunos. Em todos os estudos, a tutoria entre pares, atuou como variável de intervenção das mudanças.

Apenas um artigo utilizou-se da tutoria "Classwi- 
Quadro 1 - Artigos selecionados, após aplicação dos critérios de inclusão e exclusão.

\begin{tabular}{|c|c|c|c|}
\hline Autor (Ano). Título & Objetivo & Materiais e Métodos & Resultados/Conclusão \\
\hline $\begin{array}{l}\text { Klavina A. et. al. } \\
\text { (2014). } \\
\text { Cooperative } \\
\text { oriented learning in } \\
\text { inclusive physical } \\
\text { education } 20\end{array}$ & $\begin{array}{l}\text { Avaliar o impacto da } \\
\text { tutoria entre pares, } \\
\text { sobre a interação entre } \\
\text { os alunos com e sem } \\
\text { deficiência em aulas } \\
\text { de educação física } \\
\text { inclusiva. }\end{array}$ & $\begin{array}{l}\text { Participaram } 04 \text { alunos com deficiências } \\
\text { e } 37 \text { alunos sem deficiência, que foram } \\
\text { treinados como tutores, pertencentes à } \\
03 \text { colégios da Suécia. Realizou-se } 43 \\
\text { filmagens de aulas de educação física } \\
\text { inclusiva e analisadas pelo Computerized } \\
\text { Evaluation o flnteractions in Physical Edu- } \\
\text { cation (CEPI-PE). Posteriormente, realizou- } \\
\text { se entrevistas semi-estruturadas com os } \\
\text { alunos, professores de educação física e } \\
\text { diretores dos colégios participantes. }\end{array}$ & $\begin{array}{l}\text { A interação entre alunos com e } \\
\text { sem deficiência aumentou durante } \\
\text { o período de intervenção da tutoria. } \\
\text { Observou-se um aumento de ativi- } \\
\text { dades independentes realizadas nas } \\
\text { aulas de educação física pelos alunos } \\
\text { com deficiência. Os resultados confir- } \\
\text { mam o impacto positivo de mudança e } \\
\text { melhoria na relação entre os pares. }\end{array}$ \\
\hline $\begin{array}{l}\text { Klavina, A.; Block, } \\
\text { M. E (2008). } \\
\text { The effect of peer } \\
\text { tutoring on interac- } \\
\text { tion behaviors in } \\
\text { inclusive physical } \\
\text { education }{ }^{21}\end{array}$ & $\begin{array}{l}\text { Avaliar o efeito da tuto- } \\
\text { ria entre pares sobre os } \\
\text { comportamentos físicos, } \\
\text { de interação social e } \\
\text { instruções (mediada } \\
\text { pelo professor e pelos } \\
\text { pares e voluntária dos } \\
\text { pares) entre estudantes } \\
\text { da escola primária com } \\
\text { deficiências graves e } \\
\text { múltiplas e colegas sem } \\
\text { deficiência. }\end{array}$ & $\begin{array}{l}\text { Participaram deste estudo } 03 \text { estudantes } \\
\text { com deficiências múltiplas e } 09 \text { colegas } \\
\text { tutores de suas respectivas turmas, de } \\
\text { duas escolas elementares dos EUA. } \\
\text { Foram filmadas e analisadas } 46 \text { aulas } \\
\text { de educação física inclusiva, com } 30 \\
\text { minutos de duração cada, sendo que os } \\
\text { alunos com deficiência usavam micro- } \\
\text { fones para permitir a análise de interação } \\
\text { nas atividades. Os pares tutores foram } \\
\text { treinados em } 3 \text { sessões consecutivas de } \\
30 \text { min, sendo as filmagens analisadas } \\
\text { através do CEPI-PE. }\end{array}$ & $\begin{array}{l}\text { Verificou-se que durante a condição } \\
\text { de instrução mediada e voluntária } \\
\text { dos pares, os comportamentos de } \\
\text { instrução e de interação física entre } \\
\text { os alunos com e sem deficiência } \\
\text { aumentaram, e as interações sociais } \\
\text { mantiveram-se baixas. Os dados de } \\
\text { tempo de engajamento nas atividades } \\
\text { aumentaram para todos os alunos } \\
\text { com deficiências em todas as sessões } \\
\text { de intervenção, enquanto que as inter- } \\
\text { ações entre os alunos com deficiência } \\
\text { e os professores Diminuíram. }\end{array}$ \\
\hline $\begin{array}{l}\text { Wiskochil, B. et. al. } \\
\text { (2007) } \\
\text { The effects of } \\
\text { trained peer } \\
\text { tutors on the } \\
\text { physical } \\
\text { education of } \\
\text { children who are } \\
\text { visually impaired }{ }^{22}\end{array}$ & $\begin{array}{l}\text { Avaliar o efeito de co- } \\
\text { legas tutores treinados } \\
\text { e não treinados no } \\
\text { aprendizado de crianças } \\
\text { com deficiência visual, } \\
\text { nas aulas de educação } \\
\text { física. }\end{array}$ & $\begin{array}{l}\text { Participaram deste estudo } 04 \text { alunos } \\
\text { com deficiência visual (dois com baixa } \\
\text { visão e dois que eram cegos) e } 04 \text { tutores } \\
\text { de mesma idade e sexo dos tutorados, } \\
\text { participantes de aulas de educação física } \\
\text { inclusiva. Os instrumentos incluíram uma } \\
\text { versão modificada do ALT-PE para análise } \\
\text { do comportamento motor e questionários } \\
\text { aplicados nos tutorados, tutores e profes- } \\
\text { sores. }\end{array}$ & $\begin{array}{l}\text { Constatou-se um aumento médio } \\
\text { de } 20,8 \% \text { na performance motora, } \\
\text { analisadas através do ALT-PE em habi- } \\
\text { lidades abertas e fechadas. Os tutores } \\
\text { treinados foram mais eficazes do que } \\
\text { colegas tutores não treinados para } \\
\text { auxilio dos alunos com deficiência } \\
\text { visual nas aulas de educação física. }\end{array}$ \\
\hline $\begin{array}{l}\text { Ward, P.; Ayvazo, } \\
\text { S. (2006). } \\
\text { Classwide } \\
\text { peer tutoring in } \\
\text { physical education: } \\
\text { assessing its Effects } \\
\text { with kindergartners } \\
\text { with autism } \\
\text { w }^{3}\end{array}$ & $\begin{array}{l}\text { Avaliar os efeitos da } \\
\text { tutoria entre os pares } \\
\text { como estratégia de } \\
\text { inclusão para crianças } \\
\text { com autismo nas aulas } \\
\text { de educação física. }\end{array}$ & $\begin{array}{l}\text { Estudo realizado em uma classe de } \\
\text { jardim de infância, sendo que } 04 \text { com } \\
\text { autismo participaram da pesquisa. Foram } \\
\text { analisadas: nível de engajamento e o } \\
\text { trabalho realizado pelos alunos em } 26 \\
\text { aulas. Observadores foram treinados para } \\
\text { verificar as explicações sobre cada tarefa } \\
\text { e seus elementos críticos. }\end{array}$ & $\begin{array}{l}\text { Os resultados demonstraram melhora } \\
\text { no nível de participação e nas habili- } \\
\text { dades de duas das crianças autistas, } \\
\text { mas sugerem mais pesquisas para } \\
\text { fundamentar a tutoria como estratégia } \\
\text { de ensino para crianças autistas. }\end{array}$ \\
\hline
\end{tabular}

$d e^{\prime \prime}$, que trata-se de uma versão particular da tutoria entre pares, onde a classe inteira de alunos está envolvida na função reciproca de tutor e tutorado. Os demais artigos utilizaram-se da tutoria aos pares, onde os tutores são definidos através de critérios pré estabelecidos, sempre sendo participantes da mesma aula de educação física dos alunos com deficiência. Todos os alunos selecionados como tutores passaram por sessões de treinamento para serem definidos para essa função.

Apenas um dos artigos adotou uma abordagem mista, para interpretação dos resultados, utilizando-se tanto da abordagem quantitativa, quanto da abordagem qualitativa para análise dos resultados. Os outros três artigos selecionados fizeram uso somente da abordagem quantitativa.

A pesquisa realizada por Klavina e colaboradores ${ }^{20}$ teve como objetivo de avaliar as interações sociais dos alunos com deficiências físicas leves e moderadas em aulas de educação física. Os dados foram coletados através da filmagem e análise de 43 aulas de educação física em duas condições, dirigidas pelo professor e mediada pelos pares. Para análise das filmagens foi utilizado o Protocolo Informatizado de Avaliação das Relações em Educação Física, um software de computador que avalia as interações em variáveis classificadas em três sub-categorias: instrucional, física e interações sociais. ${ }^{24}$ Também foi realizada neste estudo entrevistas com análise de conteúdo para fornecer informações complementares aos resultados quantitativos.

O mesmo protocolo acima foi utilizado na pesquisa realizada por Klavina e Block. ${ }^{21}$ Neste estudo foi avaliado o efeito da tutoria sobre os comportamentos físico, instrucional e de interação social entre estudantes da escola primária com deficiências múltiplas e seus pares sem deficiências em aulas de educação física, em três condições de ensino: dirigida pelo professor, mediada 
pelos pares, e com apoio voluntário dos pares em duas escolas. Foram utilizados nove tutores dos pares selecionados a partir da participação nas mesmas aulas de educação física dos alunos com deficiências e por recomendação dos professores. Foram filmadas 46 aulas de educação física dividas pelo tempo, nas três condições citadas acima.

O estudo realizado por Wiskochil e colaboradores ${ }^{22}$ objetivando avaliar o efeito da tutoria de pares no tempo de aprendizado acadêmico nas aulas de educação física - ALT-PE, de quatro crianças com deficiência visual coletados através da filmagem de aulas de educação física, divididas em duas fases: base (quatro a seis aulas) e de intervenção (seis a oito aulas), comparando a performance dos alunos com deficiência nas fases de base e de intervenção. O número de aulas variou porque as coletas foram realizadas em diferentes escolas. Medidas de percentagem do ALT-PE foram calculadas para cada participante em cada aula. Posteriormente, foram realizadas as análises visuais das gravações de cada participante para avaliar se mudanças foram apresentadas nos padrões da performance e se, estas mudanças ocorreram em função da intervenção dos tutores. Também foram comparados os resultados de ALT-PE na execução de habilidades motoras abertas e fechadas.

No estudo realizado por Ward e Ayvazo, ${ }^{23}$ foi avaliado o efeito da tutoria Classwide (CWTP) como estratégia de inclusão para crianças com autismo nas aulas de educação física, através da análise da participação e desempenho realizado pelos alunos com autismo, quando comparados com seus pares de mesma idade no desempenho de tarefas motoras consideradas como próprias para a idade. Foram gravadas e analisadas 26 aulas de educação física, que consistia numa breve introdução, pratica de atividade física moderada por 10 minutos, seguidas com demonstração das atividades alvo a serem realizadas e da prática da atividade demonstrada por exatos 2 minutos, marcados por uma música.

Todos os estudos selecionados nesta revisão utilizaram-se da filmagem como método de análise dos dados. Ressalta-se que todos os avaliadores envolvidos nos estudos selecionados nesta revisão tiveram que atingir uma percentagem de concordância inter-observador e entre observadores para estabelecer os critérios de confiabilidade.

A inclusão do aluno com deficiência representa atualmente um desafio, desde a modalidade de educação infantil ao ensino superior, tanto de instituições públicas, quanto de privadas ${ }^{25}$. Foi verificado que a inclusão de pessoas com deficiência pode ser iniciada na pré-escola ou escola primária nas quais os objetivos educacionais são cognitivos, sociais, desenvolvimento motor e comportamento adaptativo. ${ }^{20}$ Entretanto, mesmo em participantes com idade escolar um pouco mais avançada, a tutoria em pares pode ser empregada de maneira positiva aos alunos. ${ }^{23}$

A tutoria com a participação de crianças, com e sem deficiência, incentiva o aprendizado e a convivência entre elas no ambiente que dividem, ${ }^{26}$ o que vem a ser positivo para todos os envolvidos neste processo. Apesar disto, o estudo de Klavina e Block ${ }^{21}$ não concluiu que a tutoria seja eficaz quanto à interação social, embora tenha apresentado resultados favoráveis ao engajamento e interação física entre os alunos com deficiência e seus tutores.

Entre os resultados favoráveis encontrados nos estudos de Klavina e colaboradores, ${ }^{20}$ Ward e colaboradores $^{23}$ e Wiskochil e colaboradores ${ }^{22}$ verificamos que estes autores defendem que a tutoria aos pares promove a educação inclusiva através de uma conformidade organizada e projetada para que todos os alunos, com e sem deficiência, apresentem uma identidade de participação durante as aulas.

Tanto Ward e Ayvazo, ${ }^{23}$ quanto Klavina e colaboradores ${ }^{20}$ relataram que a tutoria entre pares pode contribuir com a colaboração bem-sucedida entre alunos com e sem deficiência, por aumentar suas habilidades de conversação, sentimentos de companheirismo e a sensação de pertencer aquele lugar. ${ }^{27} \mathrm{~A}$ interação social que os alunos com deficiência e seus tutores experimentam, durante as aulas de educação física, promovem aumento do companheirismo entre eles, fazendo que essa interação continue inclusive em outros ambientes, em convívio no refeitório, intervalo entre as aulas e recesso de aulas. ${ }^{28}$

Enquanto a proximidade entre um aluno com deficiência e um professor é desejável, por vezes essencial, o apoio excessivo do adulto pode ser prejudicial para um estudante com deficiência, pois pode aumentar a sua dependência à ele e, por sua vez, provocar a separação de outros colegas. ${ }^{29}$

Dentre as dificuldades encontradas para a realização das pesquisas selecionadas nesta revisão, pode-se elencar: a seleção intencional da amostra, o fato de os alunos sem deficiência serem voluntários, as características individuais dos tutores (por exemplo, experiência anterior em interações com os colegas com deficiência), a presença do pesquisador na classe de educação física inclusiva, ${ }^{21}$ a dificuldade de coleta de dados nos ambientes naturais (ambiente ativo e ambientes ruidosos), a dificuldade na gravação das interações verbais, as dificuldades burocráticas (um dos pais de alunos com deficiência não deu permissão para usar o gravador de voz), a grande diferença entre os professores, quanto à experiência de ensino com alunos com deficiência $(5$ a 30 anos), o número pequeno da amostra de estudantes com deficiência, ${ }^{20}$ a impossibilidade de generalizar os resultados para a população de crianças com a mesma deficiência dos participantes da pesquisa, devido à variação de sexo, idade e o pequeno número da amostra. ${ }^{22}$

Observou-se que são inúmeras as dificuldades encontradas pelos pesquisadores, entretanto os dados apresentados descrevem benefícios da interação de tutores de mesma faixa etária dos alunos com deficiência, assim como refere também a importância do treinamento dos professores. Apesar dos resultados serem considerados positivos, ainda não se obtém um consenso para o perfil do tutor e como a tutoria deve ser feita.

Com esta acepção, torna-se evidente a necessidade de novos estudos acerca desta temática, pois é fundamental verificar qual seriam os tipos de intervenções mais adequados de tutoria, frente às distintas deficiências e diferentes faixas etárias, para assim fazer melhor uso desta metodologia nas classes de educação física.

Ressalta-se ainda que outras variáveis devem ser abordadas em futuras pesquisas, tais como avaliar o 
comportamento de alunos com e sem deficiência após o período de intervenção da tutoria, de modo a verificar se as atitudes favoráveis caracterizam um valor duradouro, ou se não incentivadas, deixam de ser praticadas.

\section{CONSIDERAÇÕES FINAIS}

A presente revisão sistemática investigou dados presentes na literatura atual, referentes às possibilidades de cooperação de colegas de turma para favorecer a participação de alunos com deficiências em aulas de educação física. Os resultados deste estudo demonstraram que a tutoria aos pares, utilizada como um suporte pelo professor nas aulas de educação física, pode facilitar a interação entre alunos com e sem deficiência, demonstrando-se ser uma valiosa estratégia para a inclusão, pois favorece positivamente as interações entre os alunos com e sem deficiência. Fica também evidenciado que a tutoria aos pares atua positivamente no tempo de participação do aluno com deficiência nas atividades trabalhadas, tornando assim, as aulas mais efetivas quanto aos benefícios físicos e psicológicos da participação nas aulas para os alunos com deficiência. Além disso, promove ainda a educação inclusiva por meio de uma conformidade organizada e projetada para que todos os alunos, com e sem deficiência, apresentem uma identidade de participação durante as aulas, propiciando o empoderamento de alunos com deficiência através da participação nas aulas de educação física e contribuindo com a colaboração bem-sucedida entre alunos com e sem deficiência, aumentando habilidades de conversação, sentimentos de companheirismo e a sensação de pertencimento ao grupo.

Todavia, observa-se que sem a utilização de uma adequada assistência suplementar, a inclusão de alunos com deficiência na aula de educação física escolar pode não ser bem-sucedida, e ressalta-se, portanto, a necessidade de um adequado planejamento desta prática.

Os resultados encontrados por este trabalho transparecem a necessidade de realizar novos estudos que abordem especificamente os tipos de tutoria, de forma a contribuir, para uma melhora na inclusão de pessoas com deficiências no âmbito escolar, em especial nas aulas de educação física.

\section{REFERÊNCIAS}

1. Piccolo GM, Mendes EG. Contribuições a um pensar sociológico sobre a deficiência. Educ Soc 2013;34(123):459 475. doi: 10.1590/S0101-73302013000200008.

2. Bampi LNS, Guilhem D, Alves ED. Social Model: A New Approach of the Disability Theme. Rev Latino-Am Enfermagem 2010;18(4):816-823. doi: 10.1590/S0104 11692010000400022.

3. Declaração de Alma-Ata, Conferência Internacional sobre cuidados primários de saúde; 6-12 de setembro 1978; AlmaAta; USSR. Ministério da Saúde (BR). Secretaria de Políticas de Saúde. Projeto Promoção da Saúde. Declaração de Alma-Ata, 15.

4. Dantas TC, Silva JSS, Carvalho MEP. Entrelace entre gênero, sexualidade e deficiência: uma história feminina de rupturas e empoderamento. Rev Bras de Educ Espec 2014;20(4):555568. doi: 10.1590/S1413-65382014000400007.

5. Braga MMS, Schumacher AA. Direito e inclusão da pessoa com deficiência: uma análise orientada pela teoria do reconhecimento social de Axel Honneth. Sociedade e Estado 2013;28(2):375-392. doi: 10.1590/S010269922013000200010.

6. Mauerberg-decastro E, Paiva ACS, Figueiredo GA, Costa TDA, Castro MR, Campbell DF. Attitudes about inclusion by educators and physical educators: effects of participation in an inclusive adapted physical education program. Motriz: Rev Educ Fís 2013;19(3):649-661. doi: 10.1590/S198065742013000300017

7. Caiado KRM, Laplane ALF. Programa Educação inclusiva: direito à diversidade - uma análise a partir da visão de gestores de um município-polo. Educação e Pesquisa 2009;35:303315. doi: 10.1590/S1517-97022009000200006.

8. Américo BL, Carniel F, Takahashi ARW. Gestão pública da educação especial e formalismo nas políticas públicas inclusivas - o caso do Brasil. Ensaio: Avaliação e Políticas Públicas em Educação 2014;22(83):379-410. doi: 10.1590/ S0104-40362014000200006.

9. Laplane ALFD. Condições para o ingresso e permanência de alunos com deficiência na escola. Cadernos CEDES 2014;34(93):191-205. doi: 10.1590/S010132622014000200004.

10. Soler R. Educação física inclusiva: em busca de uma escola plural. Rio de Janeiro: Sprint; 2005.

11. Souza JV. Tutoria: Estratégias de Ensino para Inclusão de Alunos com Deficiência em Aulas de Educação Física. Tese (Doutorado em Educação Especial) - Universidade Federal de São Carlos, São Carlos, 2008.

12. Munster MAV. Inclusão de Estudantes com Deficiências em Programas de Educação Física: Adaptações Curriculares e Metodológicas. Revista da Sobama 2013;14(2):27-34.

13. Aguiar JC, Duarte E. Educação inclusiva: um estudo na área da educação física. Rev Bras Educ Especi 2005;11(2):223240. doi: 10.1590/S1413-65382005000200005.

14. Declaração de Jacarta. Quarta Conferência Internacional de promoção da saúde; Jacarta, Indonésia; 1997.

15. Ol J, Ha AS. Inclusion in Physical Education: A review of literature. International Journal of Disability, Development and Education 2012;59(3):257-281. doi: 10.1080/1034912X.2012.697737.

16. Castro EM. Tutores: a inclusão parceira. Revista Adapta; 2005.

17. MauerbergdeCastro EM. Atividade Física Adaptada. Tecmedd. Ribeirão Preto; 2005.

18. Mauerberg-decastro EM. Atividade Física Adaptada. 2. Ed. Ribeirão Preto Novo Conceito; 2011.

19. De-la-torre-ugarte-guanilo MC, Takahashi RF, Bertolozzi MR. Revisão sistemática: noções gerais. Rev Esc Enferm 2011;45(5):1260-6. doi: 10.1590/S0080-62342011000500033.

20. Klavina A, Jerlinder K, Kristén L, Hammar L, Soulie T. Cooperative oriented learning in inclusive physical education. European Journal of Special Needs Education 2014;29(12):119-134. doi: 10.1080/08856257.2013.859818

21. Klavina A, Block ME. The effect of peer tutoring on interaction behaviors in inclusive physical education. Adapted physical activity quarterly 2008;25(5):132-158.

22. Wiskochil B, Lieberman LJ, Houston-Wilson C, Petersen S. The Effects of Trained Peer Tutors on the Physical Education of Children Who Are Visually Impaired JVIB 2006;101(6):1-18.

23. Ward P, Ayvazo S. Class wide Peer Tutoring in Physical Education: Assessing Its Effects With Kindergartners With Autism. Adapted Physical Activity Quarterly 2006;23(3):233- 
244. doi: 10.1123/apaq.23.3.233.

24. Klavina A. Development and Initial Validation of the Computerized Evaluation Protocol of Interactions in Physical Education. Measurement in Physical Education and Exercise Science 2011;15(1):26-46. doi: 10.1080/1091367X.2011.537868.

25. Duarte ER, Rafael CBS, Filgueiras JF, Neves CM, Ferreira MEC. Estudo de caso sobre a inclusão de alunos com deficiência no Ensino Superior. Rev Bras Educ Espec 2013;19(2):289-300. doi: 10.1590/S1413-65382013000200011.

26. Wolery M, Martin CG, Schroeder C, Huffman K, Venn ML, Holcombe A, Brookfield J, Fleming LA. Employment of Educators in Preschool Mainstreaming: A Survey of General Early Educators. Journal of Early Intervention 1994;18:64-
77. doi: $10.1177 / 105381519401800106$.

27. Hughes C, Rung LL, Wehmeyer ML, Agran M, CopelandSR, Hwang B. Self-prompted Communication Book Use to Increase Social Interaction among High School Children. The journal of the association for persons with severe hándicap 2000; 25(3):153-166. doi: 10.2511/rpsd.25.3.153.

28. Goodwin DL, Watkinson EJ. Inclusive Physical Education from the Perspective of Students with Physical Disabilities. Adapted physical activity quarterly 2000;17:144-160. doi: 10.1123/apaq.17.2.144

29. Giangreco MF, Edelman SW, Luiselli TE, MacFarland SZC. Helping or hovering? Effects of instructional assistant proximity on students with disabilities. Exceptional Children 1997;64:7-18. doi: 10.1177/001440299706400101.

Como citar: SCHULLER, Juliana Aparecida de Paula et al. Tutoria nas aulas de Educação Física inclusiva: uma revisão sistemática. Cinergis, Santa Cruz do Sul, v. 17, n. 3, set. 2016. ISSN 2177-4005. Disponível em: <https://online.unisc.br/seer/ index.php/cinergis/article/view/8134>. Acesso em: 11 out. 2016. doi:http://dx.doi.org/10.17058/cinergis.v17i3.8134. 